\title{
Determination of Aerobic Performance in Youth Soccer Players: Effect of Direct And Indirect Methods
}

\author{
by \\ Wonder Passoni Higino ${ }^{1}$, Alex de Souza Sorroche ${ }^{2}$, \\ Pedro Guilherme de Mattos Falqueiro², Yuri Cristina Suzuki Lima3, \\ Camila Lie Higa ${ }^{4}$
}

This study was conducted to correlate and compare values for variables determined in indirect tests with the values determined directly in youth soccer players. The study subjects were 27 youth soccer players (age $16.77 \pm 0.75$ years; body mass $63.29 \pm 7.37 \mathrm{~kg}$; body height $174.14 \pm 8.46 \mathrm{~cm}$ ) playing in the basic categories of a first division team at the regional level of Brazilian soccer. Each subject was evaluated with the following tests: a) a treadmill test to directly determine values of $\mathrm{VO}_{2 \max }$ and Vamax (Treadmill); b) an indirect Shuttle Run Test (SRT); c) an indirect Carminatti's test (TCar). VO2max showed significantly different values in the Treadmill and the SRT (59.21 \pm 5.88 and $50.67 \pm 3.58 \mathrm{ml} \cdot \mathrm{kg}^{-1} \cdot \mathrm{min}^{-1}$, respectively). Similarly, values obtained for VPeak in the treadmill test and for Vamax in TCar were different from values for SRT $V_{\text {Peak }}\left(15.01 \pm 1.10,14.92 \pm 0.87\right.$ and $12.64 \pm 0.62 \mathrm{~km} \cdot h^{-1}$, respectively). A correlation analysis showed a moderate relationship between values for $V_{\text {Peak }} T C$ ar and $V O_{2 m a x}$ determined on a treadmill $(r=0.46)$ and Vamax determined on a treadmill $(r=0.54)$. The analysis also showed a high correlation between values of $V \mathrm{O}_{2 \max }$ determined on the treadmill and VO2max evaluated in the SRT $(r=0.69)$, as well as VPeak determined in the SRT and $V O_{2 m a x}$ tested on the treadmill $(r=0.71)$, as well as between $V_{\text {Peak }}$ determined in the SRT and VO2max evaluated on the treadmill $(r=0.77)$. We concluded that the SRT underestimated values of $V O_{2 \max }$ and Vamax. Additionally, $V$ peak $T C a r$ showed no difference compared to Vamax, although it did show a low correlation with it. In addition the SRT, even with high correlations, did not seem to be a great predictor of aerobic fitness in youth soccer players.

Key words: treadmill test, shuttle run test, Carminatti's test, maximal oxygen uptake, youth soccer.

\section{Introduction}

The complexity of soccer, especially from a physiological point of view, can be fully appreciated when one considers the main actions performed by players during a game. Di Salvo et al. (2007) reported that during a match, soccer players experienced short and intense periods of great effort characterized by periods of anaerobic metabolism; however, aerobic metabolism predominated during the recovery periods.
The value of $\mathrm{VO}_{2 \max }$ is the index most widely used for measuring aerobic fitness of soccer players, and can be determined in both laboratory and field tests. However, since soccer involves intermittent and sudden changes in a player's direction, the nature of tests used to determine $\mathrm{VO}_{2 \max }$ in the laboratory conditions can be quite different from the actions observed during actual play (Drust et al., 2007).

\footnotetext{
1 - Instituto Federal de Educação, Ciência e Tecnologia do Sul de Minas Gerais - Campus Muzambinho - Curso de Educação Física.

2 - Universidade Estadual Paulista - Campus Bauru - curso de Educação Física.

3 - Centro Universitário Católico Salesiano Auxilium - Campus Lins - Curso de Educação Física.

4 - Universidade Estadual Paulista - Campus Rio Claro - Departamento de Educação Física.
} 
For this reason, several field tests have been suggested to verify aerobic fitness of soccer players. Among the most widely used field tests, we studied those which required continuous activities such as the Shuttle Run Test (SRT) suggested by Léger and Lambert (1982), and also tests requiring intermittent exercise such as the Carminatti's test (TCar) proposed by Carminatti et al. (2004).

Reports concerning correlations between the results of the SRT and TCar tests and the results of laboratory tests, as well as the variables measured in the two types of tests (field and laboratory) are scarce and controversial (Aziz et al., 2005; Denadai et al., 2002; Silva et al., 2011; St Clair Gibson et al., 1998). Thus, we performed the present study to correlate and compare the results of variables measured in indirect and field tests (SRT and TCar) with the results of the same variables evaluated in laboratory tests in a group of youth soccer players.

\section{Material and Methods}

\section{Subjects}

This study included 27 healthy youth male soccer players (age $16.77 \pm 0.75$ years) who were members of a first division team playing at the regional level of Brazilian soccer. All study participants had been systematically training for at least one year. The study protocol was approved by the Ethics Committee for Research at the Catholic University Center Salesian Auxilium (protocol - 452/2012). All participants and their parents were fully informed about the study's procedures and potential risks, and they provided a signed informed consent form.

\section{Measures}

Determination of body mass and body height

Each participant's height $(\mathrm{H})$ was measured using a wall stadiometer (SUNNY®) with accuracy of $0.1 \mathrm{~cm}$, and body mass (BM) was determined using a digital scale (TANITA TBF $305 \circledR)$ with accuracy of $0.1 \mathrm{~kg}$.

Determinations of $\mathrm{VO}_{2 \max }$ and $V a_{\max }$

Values of $\mathrm{VO}_{2 \max }$ and Vamax were determined using an incremental and intermittent testing protocol involving the use of a motorized treadmill (Imbramed Millenium Super ATL) maintained at a $1 \%$ incline. After a warm-up, the test was initiated at a speed of $10 \mathrm{~km} \cdot \mathrm{h}^{-1}$, which was increased by $1 \mathrm{~km} \cdot \mathrm{h}^{-1}$ every $3 \mathrm{~min}$ (corresponding to one stage) until volitional exhaustion.

Values of $\mathrm{VO}_{2 \max }$ were determined using a gas analyzer (METALYSER 3B - CORTEX®) which allowed data to be collected on a breath by breath basis. Values for the final $30 \mathrm{~s}$ of each stage were averaged. The highest $\mathrm{VO}_{2}$ value obtained during the last $30 \mathrm{~s}$ of each stage was recorded as $\mathrm{VO}_{2 \max }$. The velocity associated with $\mathrm{VO}_{2 \max }(\mathrm{Va} \max )$ was the lowest exercise intensity at which $\mathrm{VO}_{2 \max }$ was obtained. If the intensity of $\mathrm{VO}_{2 \max }$ was not sustained for at least $1 \mathrm{~min}$, the intensity at the previous stage was recorded as Vamax (Silva et al., 2011).

Shuttle Run Test (SRT)

The actual SRT was preceded by a standardized warm-up, stretching and familiarization with the protocol. The test was conducted on a soccer field which had two cones placed $20 \mathrm{~m}$ apart. The player was required to run back and forth continuously in the $20 \mathrm{~m}$ path. The player was prompted to change running speeds by an electronic device which emitted sounds at regular intervals and recorded the player's running speed. The initial running speed of 8.5 $\mathrm{km} \cdot \mathrm{h}^{-1}$ was increased by $0.5 \mathrm{~km} \cdot \mathrm{h}^{-1}$ every minute until the player voluntarily claimed exhaustion.

The player's speed during the final stage was used to calculate peak velocity (VPeak) using the following equation (Denadai et al., 2002):

$$
\text { VPeak }=\mathrm{a}+(0.5 \times \mathrm{b}) / 60
$$

where $\mathrm{a}=$ the player's velocity during the last completed stage and $b=$ the time achieved in the last completed stage.

The result obtained from the above calculation was used in the following equation to predict a value of $\mathrm{VO}_{2 \max }$ in units of $\mathrm{ml} \cdot \mathrm{kg}^{-1} \cdot \mathrm{min}^{-1}$ (Léger et al., 1988):

$$
\begin{gathered}
\mathrm{VO}_{2 \max }\left(\mathrm{ml} \cdot \mathrm{kg}^{-1} \cdot \mathrm{min}^{-1}\right)=31.025+3.238 \mathrm{X}- \\
3.248 \mathrm{~A}+0.1536 \mathrm{AX}
\end{gathered}
$$

where $X=$ peak velocity $\left(V_{\text {peak }}\right)$ in $\mathrm{km} \cdot \mathrm{h}^{-1}$ and $\mathrm{A}=$ age in years.

\section{Carminatti's test (TCar)}

The TCar was preceded by familiarization with the protocol, a standardized warm-up and stretching. The TCar is a maximal incremental test 
of a staggered intermittent type and has multiple stages of $90 \mathrm{~s}$ duration. The player performs 5 repetitions of shuttle run exercises consisting of 12 $\mathrm{s}$ of running (variable distances) interspersed with $6 \mathrm{~s}$ of walking $(\sim 5 \mathrm{~m})$. The pace is dictated by an audio signal issued at regular intervals of $6 \mathrm{~s}$. The signal determines the running speed to be attained during shifts between parallel lines marked on the ground and also marked by cones. The initial test speed of $9 \mathrm{~km} \cdot \mathrm{h}^{-1}$ (initial distance of $15 \mathrm{~m}$ ) is increased by $0.6 \mathrm{~km} \cdot \mathrm{h}^{-1}$ at each stage until the participant voluntarily indicates exhaustion while attempting to complete successive stages 1 $\mathrm{m}$ greater than the initial distance (Carminatti et al., 2004).

\section{Procedures}

All testing procedures were performed in a two-week period during which the participants attended the laboratory, the athletic track and the soccer field of Physical Education Unisalesiano Lins four different times.

At first, the players attended the laboratory, where anthropometric measurements were recorded and an intermittent progressive treadmill test to voluntary exhaustion was performed. Additionally, each participant's height, body mass, aerobic power, $\mathrm{VO}_{2 \max }$ and speed associated with $\mathrm{VO}_{2 \max }(\mathrm{Vamax})$ were evaluated. The second and third rounds of testing were conducted outside the laboratory on a soccer field and performed in random order. During these visits, the volunteers completed the Shuttle Run Test (SRT) and Carminatti's Test (TCar).

To avoid fatigue, the visits were scheduled with a 2 day interval between following tests. Additionally, the players were advised not to engage in strenuous physical activities or ingest alcoholic or caffeinated beverages at least 24 hours before each testing period. They were also required to get a good night's sleep and to consume a light meal at least $2 \mathrm{~h}$ prior to testing.

Prior to all physical tests, the participants performed a standardized warm-up and stretching which lasted $\sim 10 \mathrm{~min}$. The first $5 \mathrm{~min}$ were used for the warm-up at $50-60 \%$ of the participant's maximum heart rate $\left(\mathrm{HR}_{\max }\right)$ as determined by the Karvonen formula (Camarda et al., 2008). The second 5 min consisted of stretching the main muscle groups to be utilized in the test.

\section{Statistical Analysis}

Data were analyzed using descriptive statistics and results are expressed as mean \pm standard deviation (SD). The normal distribution of data was tested using the Shapiro-Wilk test. Normally distributed data were analyzed as follows: a) the Student's $t$ test was used to compare values obtained for $\mathrm{VO}_{2 \max }$ as determined directly using the treadmill test with $\mathrm{VO}_{2 \max }$ values determined indirectly with the Shuttle Run Test, b) analysis of variance (one-way ANOVA) with the Tukey's post-hoc test was used to compare peak velocities determined using the treadmill and Shuttle Run Tests (SRT) with those determined using the Caminatti's Test (TCar), c) the Pearson's product moment coefficient was used to verify possible correlations between values determined using indirect tests with those measured directly using laboratory tests. All statistical analyses were performed using SPSS Version 19 and $p$-values $<0.05$ were considered statistically significant.

\section{Results}

The variables examined in this study, including their means, SD and coefficients of variation are shown in Table 1.

The results of $\mathrm{VO}_{2 \max }$ are presented in Table 1 , and indicate significant differences between values obtained in the SRT and those in the treadmill test.

The values for speeds and maximal heart rate achieved in the direct and indirect tests are shown in Figures 2 and 3, respectively. There were significant differences between the values obtained from treadmill tests and those obtained from the TCar with respect to peak velocity in the SRT. There were also significant differences between the values obtained in all the tests with respect to the maximal heart rate.

Figures 4 and 5 indicate that almost all values obtained from indirect and field tests showed moderate to high correlations with values determined in laboratory conditions.

\section{Discussion}

The main objective of this study was to determine correlations between values of different variables obtained using indirect field tests specific to soccer and corresponding values obtained directly in laboratory conditions. We found differences in the values of $\mathrm{VO}_{2 \max }$ as determined directly and indirectly (SRT), as well 
as lower peak velocities in the SRT compared to peak velocities in the treadmill test and TCAR. We also found moderate to high correlations between values determined in the SRT and TCar, respectively, with corresponding values determined directly in the treadmill test. Reilly et al. (2000) reported that $\mathrm{VO}_{2 \max }$ was one of the variables most often used to physiologically characterize soccer players and this variable showed values ranging between 55 and $68 \mathrm{ml} \cdot \mathrm{kg}^{-}$ ${ }^{1} \cdot \mathrm{min}^{-1}$. This variation is primarily due to inherent differences in the tactical positions taken during a match (Reilly et al., 2000) and the fitness level of the player (Wells et al., 2012).

Table 1: Characteristics of study participants: mean value, standard deviation (SD) and coefficient of variation (CV in \%).

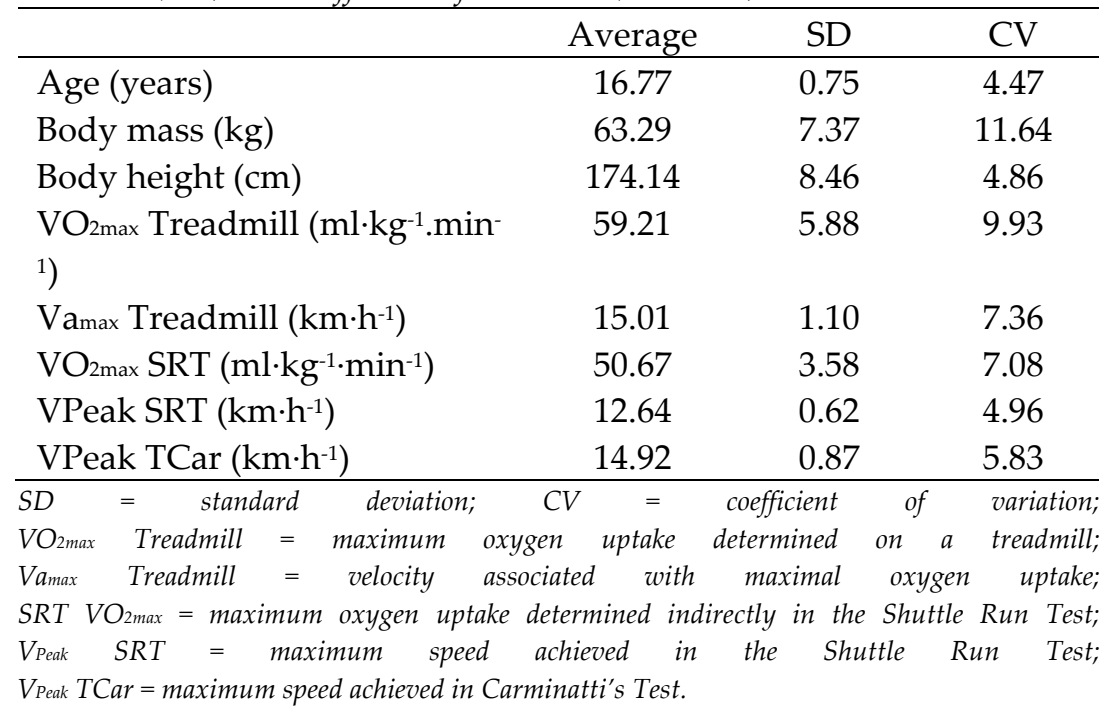

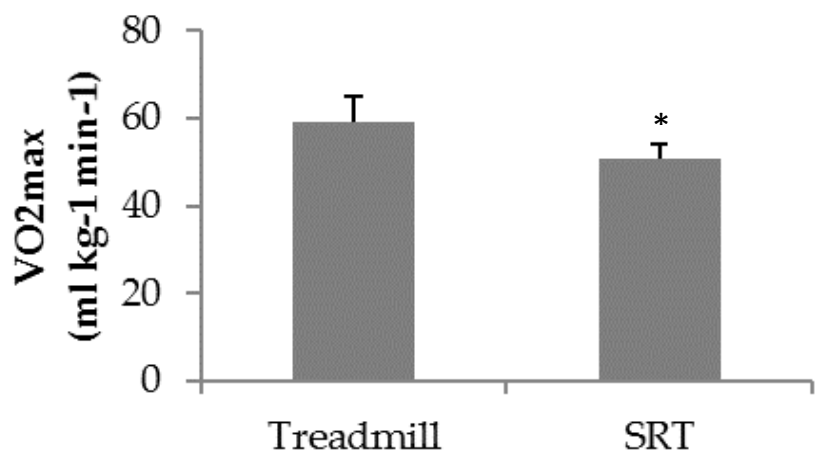

Figure 1: Maximal oxygen uptake $\left(\mathrm{VO}_{2 \max }\right)$ determined directly (Treadmill) and indirectly with the Shuttle Run Test (SRT). *Significant difference with respect to Treadmill results; $p \leq 0.05$. 


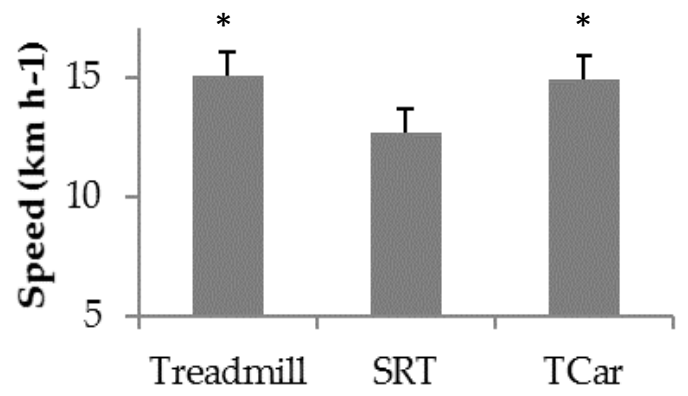

Figure 2: Maximum speeds achieved in direct tests (Vamax) and indirect tests

(velocity peak in the Shuttle Run Test - VPeak SRT and peak velocity in Carminatti's Test - VPeak TCar). *Significant difference with respect to $\mathrm{V}_{\text {peak }}$ in the SRT; $p \leq 0.05$.

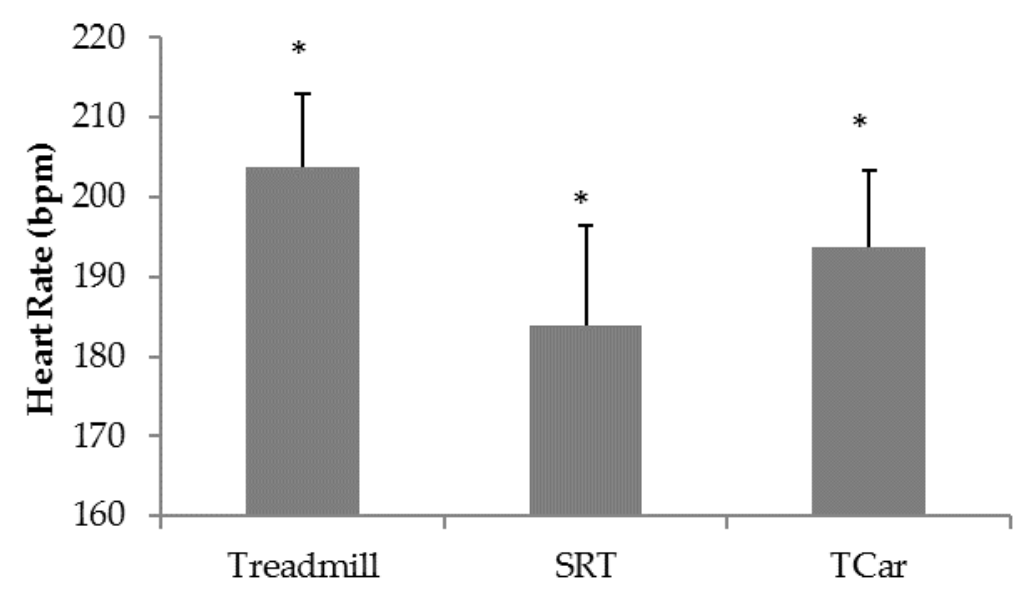

Figure 3: Maximum heart rate achieved in direct (treadmill) and indirect tests (Shuttle Run Test - SRT and Carminatti's Test - TCar). *Significant difference with respect to other tests; $p \leq 0.05$. 

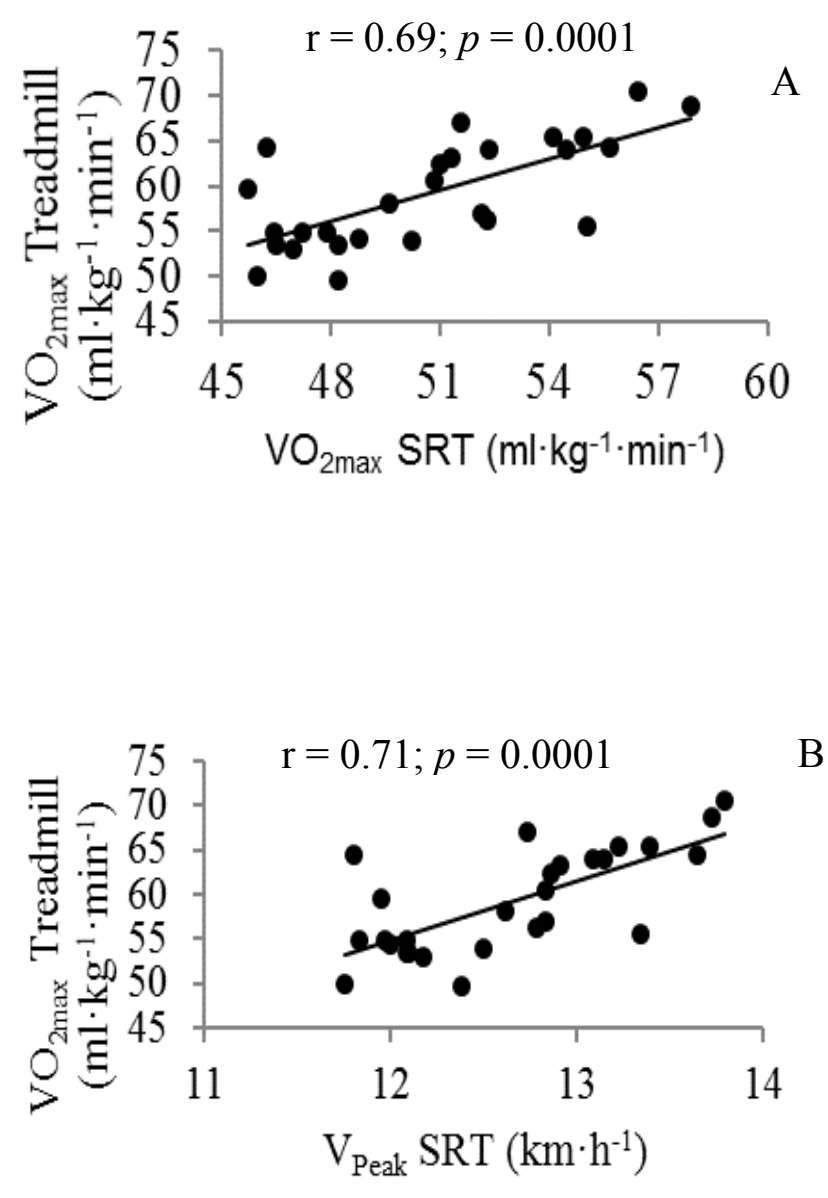

B

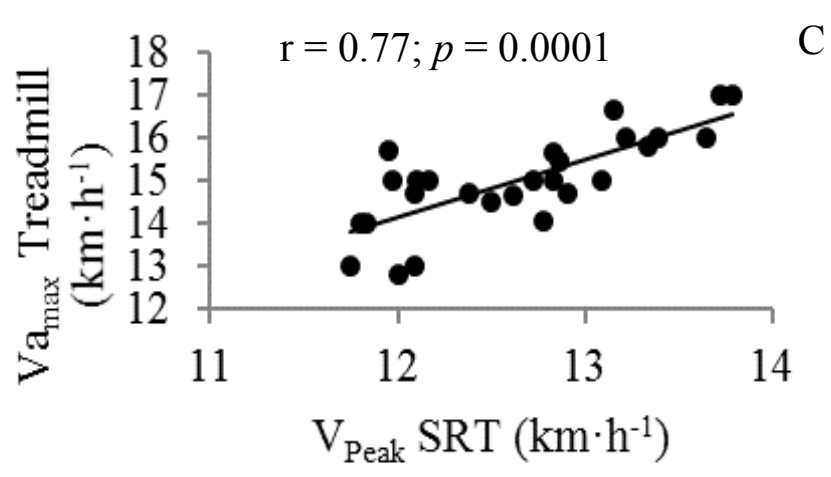

Figure 4: Correlation between maximum oxygen uptake determined directly on a treadmill $\left(\mathrm{VO}_{2} \max\right.$ Treadmill) and the value determined indirectly in the Shuttle Run Test $\left(V O_{2 m a x} S R T\right)(A)$. Correlation between $V_{2 m a x}$ Treadmill and maximum speed in the Shuttle Run Test ( $\left.V_{\text {Peak }} S R T\right)$ (B). Correlation between the velocity associated with $V_{2} O_{m a x}$ Treadmill (Vamax Treadmill) and $V_{\text {peak }} \operatorname{eSRT}(C) ; p \leq 0.05$. 

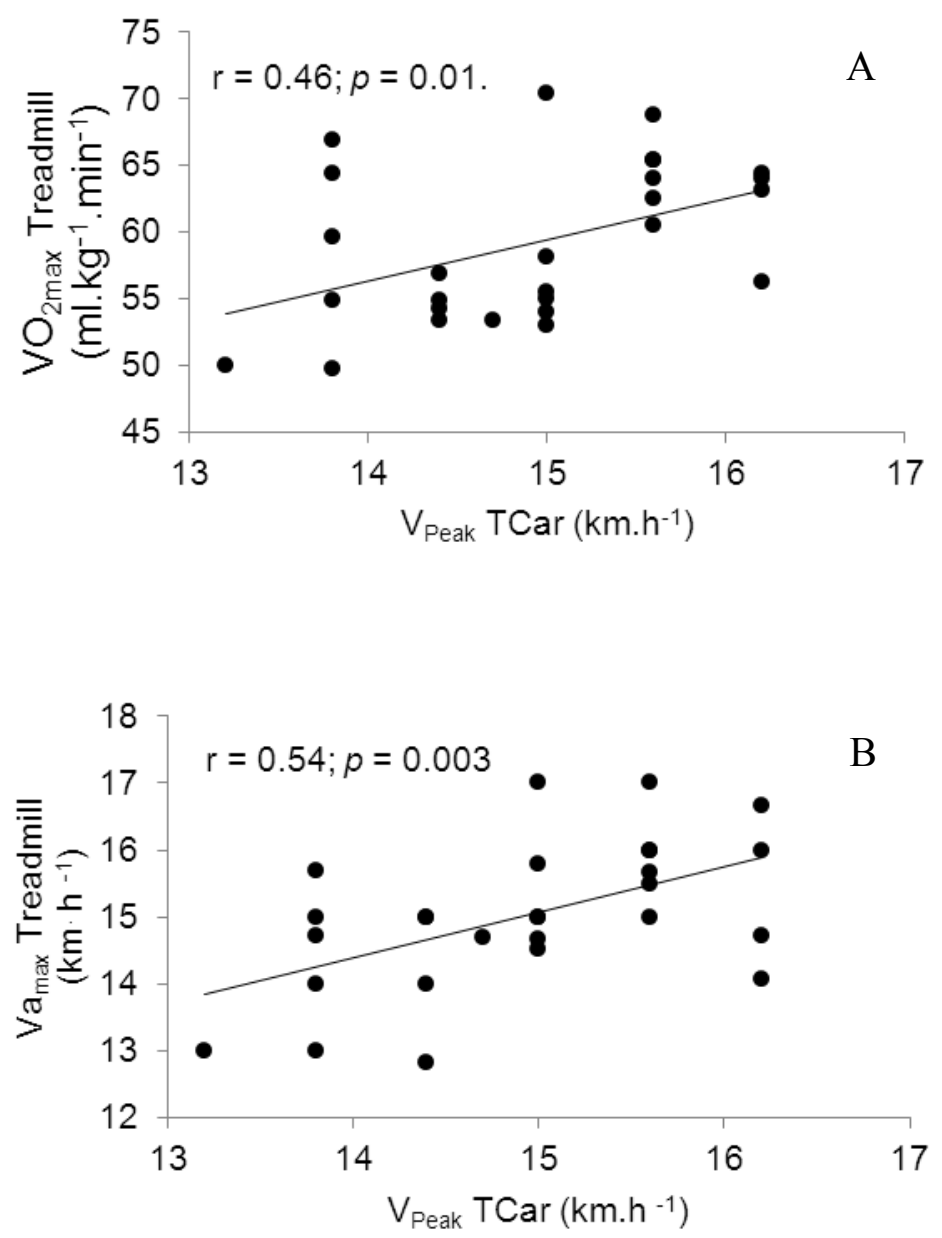

Figure 5: Correlation between maximum oxygen uptake determined directly on a treadmill $\left(\mathrm{VO}_{2 \max }\right.$ Treadmill) and the maximum speed achieved in the Carminatti's Test (VPeak TCar) (A). Correlation between the velocity associated with VO2max Treadmill (Vamax Treadmill) and VPeak TCar (B); $p \leq 0.05$.

The mean VO2max values found in this study are in line with those previously reported in the literature. Rampinini et al. (2010) found that professional soccer players with different tactical roles had similar VO2max values (mean value $\left.58.5 \mathrm{ml} \cdot \mathrm{kg}^{-1} \cdot \mathrm{min}^{-1}\right)$. Additionally, Fornaziero et al. (2009) reported similar values for this variable in semi-professional players (59 $\left.\mathrm{ml} \cdot \mathrm{kg}^{-1} \cdot \mathrm{min}^{-1}\right)$ and players in other basic categories of Brazilian soccer $\left(56 \mathrm{ml} \cdot \mathrm{kg}^{-1} \cdot \mathrm{min}^{-1}\right)$. Thus, the results of this study confirm the results of previous studies conducted with both semi-professional and well- trained professional soccer players.

Although some studies have found no differences in values of $\mathrm{VO}_{2}$ max as determined in the SRT and in laboratory conditions (Aziz et al., 2005; Edwards et al., 2003), Grant et al. (1995) reported the underestimation of $\mathrm{VO}_{2 \max }$ values when determined using predictive formulas. According to Lamb and Rogers (2007), the predictive formulas developed for measuring $\mathrm{VO}_{2 \text { max }}$ using the SRT are influenced by intrinsic factors such as the sample's size, degree of heterogeneity and other dependent variables used 
in the regression model. In the current study, we used the predictive formula developed by Léger et al. (1988), which was intended for use in populations of students, healthy adults, academic practitioners and individuals practicing sports such as basketball.

Ahmaidi et al. (1992) reported that differences in $\mathrm{VO}_{2 \max }$ as determined in different tests may be related to biomechanical factors; for instance, correct body movements are more efficient regarding energy expenditure. Making more efficient movements increases the recruitment of muscle fibers by reducing the recruitment of type II fibers and increasing the recruitment of type I fibers (Mauger and Sculthorpe, 2012). The participants of the present study were much more accustomed to performing field tests specific to soccer compared to treadmill tests. Thus, the fact that they were more familiar with tests similar to the SRT may have led them to perform movements which required less energy expenditure and maximal heart rate. Furthermore, characteristics of the SRT, such as its sudden changes in speed, direction and acceleration may have led to greater voluntary anaerobic participation (Grant et al., 1995).

A previous study by Silva et al. (2011) may help explain the difference in values obtained for Veak in the SRT and when using the TCar and Vamax (Treadmill). Those authors evaluated soccer players similar to the participants of this study and found no significant differences between values of $V_{\text {Peak }}$ in the TCar and Vamax as determined in a treadmill test. Moreover, Ahmaidi et al. (1992) and Galloti and Carminatti (2008) reported lower values for V Peak in the SRT compared to Vamax determined on a treadmill, as well as for $V_{\text {Peak }}$ evaluated in the TCar. Jones and Carter (2000) stated that the value of $\mathrm{V}_{\text {Peak }}$ in continuous tests was associated with Vamax as determined on a treadmill; however, the authors noted that this physiological index was influenced by anaerobic capacity, muscle strength and the neuromuscular ability to run at high speeds. Thus, it is likely that low values of $\mathrm{VO}_{2 \max }$ and VPeak in the SRT are associated with a higher level of effort needed to complete an incremental test without pausing, coupled with low levels of power and anaerobic capacity; all leading to a lower level of tolerance towards exercise (Galloti and Carminatti, 2008; Grant et al., 1995).
In general, the SRT appears to be highly predictive for $\mathrm{VO}_{2 \max }$ (Lamb and Rogers, 2007). Although several studies have found a low correlation between the values of $\mathrm{VO}_{2 \max }$ determined in the SRT and those determined in laboratory tests (O'Gorman et al., 2000; St Clair Gibson et al., 1998), most studies have shown the opposite (McNaughton et al., 1998; Wilkinson et al., 1999). However, according to Drust and Svensson (2005), the estimated error introduced when determining $\mathrm{VO}_{2 \max }$ using a prediction formula may lead to inaccurate results. Similarly, VPeak in the TCar correlates well with values of aerobic variables such as $\mathrm{VO}_{2 \max }$ and Vamax determined with a treadmill test (Silva et al., 2011). However, in the present study, the values of variables examined in the SRT and TCar showed strong and moderate correlations, respectively.

Castagna et al. (2006) suggested that a possible explanation for the low correlation between results of $\mathrm{VO}_{2 \max }$ and field tests designed for soccer was that the outcomes of field tests may be associated with factors other than aerobic power, such as the ability to accelerate, decelerate and quickly change directions. According to Pasquarelli et al. (2010), these abilities to some extent seem to be influenced by player's muscle power of the lower limbs, as training these abilities can positively influence player's acceleration capacity, which increases performance in progressive and multistage high intensity field tests such as the SRT and TCar test.

Although the values of $\mathrm{VO}_{2 \max }$ in our sample lie within normal values for young soccer players, the values of Vamax are below those reported in the literature (Silva et al., 2011). According to Silva et al. (2009), Vamax is the index that best describes the relationship between maximum aerobic power and running economy, as individuals with similar $\mathrm{VO}_{2 \max }$ values may have different Vamax values. This difference is explained by running economy that can be influenced by strength and anaerobic power of the athlete (Higino et al., 2011) and also has a significant effect on the outcome of our study.

Our results show that $\mathrm{VO}_{2 \max }$ determined by the formula proposed by Léger et al. (1988) underestimates values determined directly in laboratory conditions. Among the peak velocities determined in the SRT and TCar, only values of 
$V_{\text {Peak }}$ in the TCar showed no significant differences with respect to Vamax determined on a treadmill. However, both variables determined in the SRT and TCar showed low correlations with those presented in the literature. This leads us to believe that factors other than maximum aerobic power determine the outcomes of field trials such as the SRT and TCar performed with youth soccer players.

\section{Practical Implications}

Thus, as practical implications for the use of the SRT and TCar tests, it is suggested that these tests should be used with great caution with

regard to the evaluation of aerobic fitness of youth soccer players. The fact that athletes are more familiar with tests similar to the SRT may lead them to perform movements requiring less energy expenditure and maximal heart rate. Furthermore, characteristics of the SRT, such as sudden changes in speed, direction and acceleration, may also induce greater voluntary anaerobic participation (Grant et al., 1995), associated with a higher level of effort needed to complete an incremental test without pausing, coupled with low levels of power and anaerobic capacity; all leading to a lower level of tolerance towards exercise (Galloti and Carminatti, 2008; Grant et al., 1995).

Although the SRT and TCar tests are specific to soccer, they should not be considered good tools for assessing aerobic capacity of youth soccer players as they are influenced by factors other than cardiorespiratory fitness.

\section{References}

Ahmaidi S, Collomp K, Caillaud C, Préfaut C. Maximal and functional aerobic capacity as assessed by two graduated field methods in comparison to laboratory exercise testing in moderately trained subjects. Int J Sports Med, 1992; 13(3): 243-8

Aziz AR, Tan FHY, Teh KC. A Pilot Study Comparing Two Fields Tests with the Treadmill Run Test in Soccer Players. J Sport Sci Med, 2005; 4(2): 105-12

Camarda SRA, Tebexreni AS, Páfaro CN, Sasai FB, Tambeiro VL, Juliano Y, Barros TL. Comparison of Maximal Heart Rate Using the Prediction Equations Proposed by Karvonen and Tanaka. Arq Bras Cardiol, 2008; 91(5): 311-4

Carminatti LJ, Lima AES, Oliveira FR. Aerobic fitness in intermittent sports - Evidence of construct validity and results in incremental test with pause. Rev Bras Fisiol Exerc, 2004; 3(1): 1-20

Castagna C, Impellizzeri FM, Belardinelli R, Abt G, Coutts A, Chamari K, D'Ottavio S. Cardiorespiratory responses to Yo-Yo intermittent endurance test in nonelite youth soccer players. J Strength Cond Res, 2006; 20(2): 326-30

Denadai BS, Higino WP, Faria RA, Nascimento EP, Lopes EW. Validity and reproducibility of the blood lactate response during the shuttle run test in soccer players. Rev Bras Ciência Mov, 2002; 10(2): 71-8

Di Salvo V, Baron R, Tschan H, Calderon Montero FJ, Bachi N, Pigozzi F. Performance characteristics according to playing position in elite soccer. Int J Sports Med, 2007; 28(3): 222-7

Drust B, Atkinson G, Reilly T. Future perspectives in the evaluation of the physiological demands of soccer. Sport Med, 2007; 37(9): 783-805

Edwards AM, Macfayden AM, Clark N. Test performance indicators from a single soccer specific test differentiate between highly trained and recreationally active soccer players. J Sport Med Phys Fit, 2003; 43(1): $14-20$

Fornaziero AM, Leite RD, Azevedo PHSM, Dourado AC, Daros LB, Osiecki R, Stanganelli LCR. Comparative analysis of the performance of soccer players between two aerobic power tests: treadmill and Yo-Yo intermittent endurance test level 2. Rev Andal Med Deporte, 2009; 2(3): 82-6

Galloti FM, Carminatti LJ. Variable Identified In Intermittent Gradual Tests. Rev Bras Presc Fisiol Exer, 2008; 2(7): 01-17 
Grant S, Corbett K, Amjad AM, Wilson J, Aitchison T. A comparison of methods of predicting maximum oxygen uptake. Brit J Sport Med, 1995; 29(3): 147-52

Higino WP, Carreira GN, Pacheco M, Carvalho RLP. Strength training and running economy: a review of the literature. Rev Ed Física, 2011; 3: 53-62

Jones AM, Carter H. The effect of endurance training on parameters of anaerobic fitness. Sports Med, 2000; 29(6): 373-86

Lamb KL, Rogers L. A re-appraisal of the reliability of the $20 \mathrm{~m}$ multi-stage shuttle run test. Eur J Appl Physiol, 2007; 100(3): 287-92

Léger LA, Lambert J. A maximal 20m Shuttle Run Test to predict VO2max. Eur J Appl Physiol, 1982; 49(1): 112

Léger LA, Mercier D, Gadoury C, Lambert J. The multistage 20 metre shuttle run test for aerobic fitness. J Sport Sci, 1988; 6(2): 93-101

Mauger AR, Sculthorpe N. A new $\mathrm{VO}_{2}$ max protocol allowing self-pacing in maximal incremental exercise. Brit J Sport Med, 2012; 46(1): 59-63

McNaughton L, Hall P, Cooley D. Validation of several methods of estimating maximal oxygen uptake in young men. Percept Motor Skill, 1998; 87(2): 575-84

O'Gorman D, Hunter A, McDonnacha C, Kirwan JP. Validity of field tests for evaluating endurance capacity in competitive and international level sports participants. J Strength Cond Res, 2000; 14(1): 62-7

Pasquarelli BN, Santos AL, Frisselli A, Dourado AC, Stanganelli LCR. Relationship between the Bansgsbo Sprint Test with sprint, agility, lower limb power and aerobic capacity tests in soccer players. Rev Andal Med Deporte, 2010; 3(3): 87-91

Rampinini E, Sassi A, Azzalin A, Castagna C, Menaspá P, Carlomagno D, Impellizzeri FM Physiological determinants of Yo-Yo intermittent recovery tests in male soccer players. Eur J Appl Physiol, 2010; 108: 401-9

Reilly T, Bangsbo J, Franks A. Anthropometric and physiological predispositions for elite soccer. J Sport Sci, 2000; 18(9): 669-83

Silva JF, Guglielmo LGA, Floriano LT, Arins FB, Dittrich N. Fitness aerobic and repeated sprint ability in soccer: comparison between positions. Motriz, 2009; 15(4): 861-70

Silva JF, Guglielmo LGA, Carminatti LJ, Oliveira FR, Dittrich N, Paton CD. Validity and reliability of a new field test (Carminatti's test) for soccer players compared with laboratory-based measures. J Sport Sci, 2011; 29(15): 1621-8

St Clair Gibson A, Broomhead S, Lambert MI, Hawley JA. Prediction of maximal oxygen uptake from 20-m shuttle tun as measured directly in runners and squash players. J Sport Sci, 1998; 16(4): 331-5

Svensson M, Drust B. Testing soccer players. J Sport Sci, 2005; 23(6): 601-18

Wells CM, Edwards AM, Winter EM, Fysh ML, Drust B. Sport-specific fitness testing differentiates professional from amateur soccer players where $\mathrm{VO}_{2}$ max and $\mathrm{VO}_{2}$ kinetics do not. J Sport Med Phys Fitness, 2012; 52(3): 245-54

Wilkinson DM, Fallowfield JL, Myers SD. A modified incremental shuttle run test for the determination of peak shuttle running speed and the prediction of maximal oxygen uptake. J Sport Sci, 1999; 17(5): 413-9

\section{Corresponding author:}

Wonder Passoni Higino, Ph.D.

Instituto Federal de Educação, Ciência e Tecnologia do Sul de Minas Gerais, Curso de Educação Física, Rua Dinah, 75 - Bairro Jardim Canaã, CEP: 37890-000, Muzambinho, Minas Gerais/Brazil

Phone: 005535 35715818; E-mail: wonderhigino@gmail.com 